\title{
Difficulties in Translating the Language of Pirandello's Novels
}

\author{
Prof. As. Dr. Mirela Papa \\ Docente di Traduzione e Interpretariato \\ Università di Tirana \\ papamirela@yahoo.com \\ Dr. Leonard Xhamani \\ Docente di Latino \\ Università di Tirana \\ leonardxhemani@hotmail.com
}

Doi:10.5901/ajis.2013.v2n8p231

\begin{abstract}
Studying the language of Pirandello's novels is not simple. Pirandello has its own special style. When he writes he aims at writing naturally and spontaneously as if taking among friends. Thus, his way of expression is much similar to spoken language, whereas written language sometimes sounds as harsh and antiliterary. In this paper we would focus on Pirandello's diction by analyzing his loan words from different layers of the language and the way how this diction has been translated into Albanian. First, we are going to analyze dialectical words and phrases used in Pirandello's novels. He thinks that dialect can add liveliness to the language. Next, we are going to analyze words from other languages used in his novels. A writer with a wide and rich vocabulary as Pirandello cannot leave aside the use of other languages words in his novels. These words could have been suited or not to the orthography of Italian language. But there are cases when we come across entire sentences written in a foreign language. As far as other languages presence in his novels there are: French, English, Spanish and Latin. We are going to study the difficulties these words pose to be rendered into another language as well as Albanian translators strategies to convey these peculiarities of Pirandello's language into their respective languages.
\end{abstract}

Keywords: translation, literature, dialectalisms, borrowings.

\section{Introduzione allo studio}

Fare uno studio sulla lingua delle novelle di Pirandello non è facile. Pirandello ha un suo stile di scrittura particolare. Quando scrive pretende farlo con la spontaneità della parlata tra amici. II suo modo di esprimersi si avvicina di più alla lingua parlata, mentre quella scritta suona talvolta aspra e antiletteraria. In questo studio intendiamo prendere in analisi il lessico che Pirandello prende in prestito dalle altre lingue ed il modo in cui questo lessico viene tradotto nella lingua albanese. Oggetto di analisi saranno i segmenti testuali in dialetto e quelli appartenenti ad altre lingue.

\section{Caratteristiche della lingua delle novelle di Pirandello}

«Se letteratura, o meglio, tradizione letteraria ha mai fatto impedimento al libero sviluppo d'una lingua, questa più d'ogni altra è l'italiana» scrive Pirandello nei Saggi, poesie e scritti vari criticando la retorica. Ecco perché la lingua di Pirandello è antiletteraria, spontanea. Nel suo stile narrativo, espressivo e senza retorica, la prosa italiana incontra un modello a seguire, anche se a volte la sintassi del periodo si rompe o perde la sua grazia. Sono pochi gli studi concentrati sul lessico delle novelle di Pirandello e sulla provenienza di tale lessico. Pirandello come lo dimostrano i suoi scritti (Lo Vecchio-Musti 1960) sente una grande preoccupazione per la lingua italiana della sua epoca e cercherà di fare uso di tutti gli strati della lingua senza rifiutare le parole per la loro provenienza. II suo è il lessico appartenente alla lingua comune impiegata dagli italiani dell'epoca.

\subsection{Dialettalismi e traduzione}

Pirandello, educato in un ambiente della filologia romanza, si prende cura del dialetto come prezioso strumento con il 
quale arricchire la lingua italiana, per rinnovare una lingua morta e sostituire le deformità della retorica. II dialetto è capace di dare vivacità alla lingua. La ricerca della naturalità espressiva è una costante dello stile pirandelliano. Dall'analisi delle traduzioni delle novelle che costituiscono anche il corpus del nostro studio notiamo l'uso di parole in siciliano, toscano e poche parole appartenenti ad altri dialetti.

Per quanto riguarda la traduzione del dialetto occorre dire che le varietà diatopiche sono state sempre oggetto di riflessioni per la difficoltà che rappresentano nel trovare un'equivalenza linguistica. Rosa Rabadán esclude in maniera categorica la possibilità dell'equivalenza. La critica pensa che si tratti di uno di quegli argomenti problematici per i quali non esistono soluzioni soddisfacenti.

Si offrono comunque delle varie soluzioni a proposito. La prima possibilità è la traduzione del dialetto con la varietà standard o neutrale della lingua d'arrivo. Questa possibilità permette di trasferire il significato semantico del testo originale, però si produce una perdita di tono e di ricchezza espressiva, specialmente se la varietà linguistica è importante nella costruzione delle identità (Carbonell i Cortes, 1999: 92). Per alleggerire un po' questa perdita, si intromette una glossa intratestuale (del tipo «detto in dialetto») oppure extratestuale (nota a piè di pagina) in modo da informare il lettore che un personaggio parla in una determinata varietà.

La seconda possibilità consiste nella traduzione o la sostituzione del dialetto originale con un dialetto specifico della lingua d'arrivo. Catford e Julià sostengono la traduzione del dialetto come possibilità di conservare la ricchezza funzionale che esiste tra le varietà geografiche del testo d'origine. La critica esclude questa possibilità. Lefevere (1992: 69-70) ritiene che le connotazioni prodotte dalle varietà linguistiche negli utenti della traduzione di solito sono assai differenti; a questo va aggiunto il fatto che la scelta del dialetto nella lingua d'arrivo può essere condizionata da qualche approccio ideologico del traduttore. (Lefevere 1997: 78).

La terza possibilità invece prende come punto di partenza la varietà standard della lingua d'arrivo e suggerisce di sottometterla ad un processo di manipolazione introducendo vari elementi a diversi livelli (lessico-semantico e morfosintassi) per dimostrare al lettore che alcuni personaggi parlano in un modo particolare e diverso dalla forma considerata standard.

Occorre dire che nessuna delle tre opzioni non assicura la mancanza della perdita dell'espressione o del tono.

\subsubsection{Pirandello e il dialetto siciliano}

Pirandello per un lato s'accosta al dialetto della Sicilia e per un altro ne rimane sdegnosamente lontano. In Pirandello hai l'artista che mescola, non fonde, lo stile suo con quello delle sue creature, ma con la segreta speranza di spremere da quest'ultimo solo la parte sostanziosa onde farla passare nell'altro e renderlo più vitale. Nella prosa di Pirandello avverti non poche stonature (Puglisi 1968: 119). E non solo i termini, si tenga presente, stridono tra loro per la diversa fattura e provenienza, ma le costruzioni stesse della proposizione, del periodo, si trovano non di rado a disagio nella prosa pirandelliana (Puglisi 1968: 122).

Più che un accordo in Pirandello, tra la lingua dotta e quella dialettale, c'è un compromesso. Si direbbe ch'egli conceda, sì, ai suoi personaggi la facoltà di parlare nel loro dialetto, ma tosto li ferma per far passare sottobanco nel loro frasario qualche termine suo, quasi li volesse abituare a poco a poco a parlare la lingua madre (Puglisi 1968: 123).

Ecco, ci sono esclamazioni, modi di dire, propri dei Siciliani, ma, siamo sempre lì, cacciati a forza all'interno del periodo, del discorso, e che restano pertanto soli, soli, spauriti (Puglisi 1968: 126).

Cos'è che resta dunque della Sicilia nello stile di Pirandello? Non il vocabolario (...), non la sintassi ben presto superata, ma il fremito, l'ansito come direbbe lui, il nervosismo dei Siciliani che si ripercuote sul loro linguaggio, a scatti, convulso, mai pago di sé, che fa ricorso alla mimica per completarsi e che rimane, malgrado ciò, in aria il più delle volte (...) (Puglisi 1968: 119).

Ecco alcuni esempi presi dalle sue novelle.

- Quel giorno si parlava della nuova comitiva d'emigranti che la mattina dopo doveva partire per l'America. (L'altro figlio)

- Asaj dite flitej për karvanin e ri të mërgimtarëve që do të nisej për në Amerikë. (Djali tjetër, p. 146, «Jeta lakuriq», Naim Frashëri, Tiranë 1963)

Usa l'imperfetto (doveva) come fanno i siciliani per indicare un'azione che si svolgerà nel futuro. Nella traduzione, ovviamente, non si percepisce minimamente questo uso del dialetto da parte dello scrittore siciliano.

Bisogna dire, però, che è nel vocabolario dove si sente meglio l'influenza della sua terra. La forma dialettale, penetrata nella prosa di Pirandello non poteva non comprometterla: 
- Ecco laggiù la Casa della Colonna, (...). Era una catapecchia, veramente; una "roba", come i contadini di Sicilia chiamano le loro abitazioni rurali. (L'altro figlio)

- Në të vërtetë ish një kasolle, një plaçkë si i quajnë fshatarët e Siqilisë banesat e tyre. (Djali tjetër, p. 158, «Jeta lakuriq», Naim Frashëri, Tiranë 1963)

Nella traduzione la parola roba viene riportata in grassetto. Questa informazione iconica non riesce però a trasmettere al lettore il fatto che si tratta di una parola in dialetto visto che la parola albanese appartiene alla lingua standard.

- Ehi, commilitone! Picciotto! Picciotto! (Le medaglie)

- Ej, bashkëluftëtar! Djalosh! Djalosh! (Medaljet, p. 15, «Zoja prej dylli», Toena, Tiranë 2010)

Dall'Italia settentrionale proviene (attraverso la colonizzazione gallo-italica) il suffisso - ittu, alquanto diffuso nella Sicilia settentrionale, per esempio piccittu "piccolo". Nella traduzione il termine usato appartiene all'albanese standard.

- I "carusi", buttando giù il carico dalle spalle peste e scorticate, seduti su i sacchi, per rifiatare un po' all'aria, tutti imbrattati dai cretosi acquitrini lungo le gallerie o lungo la lubrica sala a gradino rotto della "buca", grattandosi la testa e guardando a quella collina attraverso il vitreo fiato sulfureo che tremolava al sole vaporando dai "calcheroni" accesi o dai forni, (...) (Il fumo)

- Shegertët, me të shkarkuar barrën që mbanin mbi shpatullat e raskapitura e të rjepura, uleshin sipër thasëve, sa për të marrë një grimë ajër; si njëri tjetri, ishin përlyer e zhgryer nëpër pellgjet e gëlqeres gjatë galerive ose duke shkelur nepër shkelëzat e thyera të shkallëve të lëmuara - ku shkisje lehtë - të "grykës" së minierës; duke kruar kokën - vështronin atë kodër, nëpërmjet afshit, si qelq, të squfurit që ngrihej përpjetë nga furrat e ndezura e fërgëllonte nëpër rrezet e diellit (Tymi, p. 53, «Jeta lakuriq», Naim Frashëri, Tiranë 1963)

Nell'esempio riportato il primo termine in dialetto (carusi) viene tradotto con l'albanese standard, mentre il secondo termine (calcheroni) viene omesso.

II sicilianismo più conosciuto di Pirandello è senza dubbio la parola lumia, la quale dà il titolo ad uno dei racconti più famosi di questo autore. Lumìe di Sicilia:

- Prese la valigetta e il sacchettino di sotto la tavola, e s'avviava per uscire quando gli venne in mente che lì, dentro il sacchetto, c'eran le belle lumie ch'egli aveva portato a Teresina dal paese. (Lumìe di Sicilia)

- Mori valixhen dhe trastën nga poshtë tryezës dhe u nis për të dalë, kur u kujtua se në atë trastë kishte ca limona kokërrmëdhenj, që i kishte sjellë nga fshati për Terezinën. (Limona Sicilie, p. 171, «Tregime e novela», Dituria, Tiranë 1999)

Anche in questo caso la traduzione del termine viene fatta con un termine che appartiene alla lingua albanese standard optando così per la prima soluzione della traduzione del dialetto offerto dalla teoria e prassi della traduzione.

In altri casi si tratta di esclamazioni, modismi propri dei siciliani, anche se a volte rimangono soli nella frase, isolati dal resto del lessico:

- $\quad$ Corna, vi cedo! - gridava. - Neanche se m'offriste i tesori di Creso; neanche se mi diceste: "Mattia, raspa qua con un piede, come fanno le galline; ci trovi tanto zolfo che diventi d'un colpo più ricco di... che dico? di re Fallari! Non rasperei, parola d'onore! (Il fumo)

- $\quad$ Asnië fije ferre, nuk lëshoj! - buçiste! - Edhe sikur të më ipni thesarët e Kresit. Edhe sikur të më thonit: Mati, gërryej këtu, me një këmbë, si bëjnë pulat; do të gjesh aq shumë squfur sa do të bëhesh menjëherë më i pasur se... se kush? Se mbreti Falar! Nuk do të gërryeja, për fjalë të nderit! (Tymi, p. 55, «Jeta lakuriq», Naim Frashëri, Tiranë 1963)

- Càzzica, che testa! - esclamò lo Zirafa. - Come parlo? V'ho detto che vi voglio i punti. C'intenderemo a lavoro finito: non ho tempo da perdere con voi. (La giara)

- Djall o djall, ç'kaptinë! - thirri Xirafa. - Sa herë duhet të flas? Të thashë se duhet ta qepësh. Merremi vesh si të mbarosh punë: nuk kam kohë të humb me ty. (Qypi, p. 10, «Jeta lakuriq», Çabej, Tiranë 1997)

In tutti i casi riportati $\mathrm{i}$ termini in dialetto vengono tradotti con termini dell'albanese standard non potendo trasmettere così nel testo tradotto lo stile dello scrittore.

\subsubsection{Pirandello e il dialetto toscano}

II dialetto toscano insieme a quello siciliano costituiscono la fonte più ricca per quanto riguarda le parole dialettali del lessico pirandelliano. L'introduzione delle parole del dialetto toscano nella lingua scritta e quella orale è molto forte dopo I'unificazione politica specialmente negli anni quando Firenze era capoluogo del regno (1865-1871). Per scrittori toscani quali: Fucini, Collodi, Martini ecc. è facile e spontaneo l'adozione delle parole o delle espressioni del dialetto toscano 
parlato. Con il passar del tempo si ridurranno le differenze tra l'italiano standard ed il toscano. Pirandello non poté evitare l'influenza esterna. Uno studio dettagliato della sua opera non può non mettere il risalto la presenza del dialetto toscano. Nella maggior parte dei casi si tratta dell'uso della varietà toscana al posto dell'equivalente dell'italiano standard.

Così per esempio:

- gli armenti (...) muglianti, dove mugliare è una variante popolare toscana del verbo mugghiare. (Filo d'aria)

- kopetë që... pëllitnin (Një fill ere, p. 194, «Nata e parë», Toena, Tiranë 2008)

- $\quad$ i paduli salsugginosi dei fiumi perduti (variante toscana di palude) (Filo d'aria)

- $\quad$ moçalet e vogla dhe të kripura të lumenjve të përhumbur (Një fill ere, p. 194, «Nata e parë», Toena, Tiranë 2008)

- Si soffiò di nuovo su le mani diacce e pestò i piedi per terra, (dove il termine diacce è una variante toscana di ghiacce che vuol dire fredde) (Lumie di Sicilia)

- hukati përsëri duart akull të ftohta dhe shkapeti këmbët për tokë (Limona Sicilie, p. 165, «Tregime e novela», Dituria, Tiranë 1999)

In tutti questi casi i segmenti dialettali sono stati tradotti con termini dell'albanese standard.

Dal punto di vista della morfologia e riguardo ai pronomi dimostrativi italiani (questo, codesto, quello) la varietà toscana «codesto» è quella che si predilige nella lingua letteraria. Nei giorni nostri si è ridotta sempre di più la zona della sua influenza. Si può affermare che solo a Toscana si nota chiaramente l'uso di cadauno dei pronomi. Pirandello toscaneggia «costà», «codesta», sono termini che in Sicilia non usa nemmeno la gente colta.

- Tu, codesti vizii te li devi levare (La rallegrata)

- Mblidhe pra e hiq dorë nga këto vese, se do gjesh belanë. (Kërcim i hareshëm, p. 140, «Jeta lakuriq», Çabej, Tiranë 1997)

- Si cambia vita da oggi. Mi sono seccato. Mi metto a fare il contadino come mio padre; e dunque tu smetterai di fare la signora costà. Via, via, tutta codesta biancheria! (Scialle nero)

- Prej sot do të rrojmë ndryshe! Jam lodhur. Do bëhem ujk, si im at; pra, ti nuk do të jesh më zonjë këtu. Flaki, flaki tutje tërë këto lecka! (Shalli i zi, p. 33, «Jeta lakuriq», Naim Frashëri, Tiranë 1963)

Accanto alle tre forme degli aggettivi dimostrativi ci sono anche le forme sostantivate usate per il soggetto «costui», «cotesti» e «colui» per il genere maschile e «costei», «cotestei» e «colei» per il genere femminile con la forma «costoro», «cotestoro» e «coloro» per il plurale. C'è da notare che «costui», «costei», «costoro» hanno assunto una connotazione peggiorativa. Anche «colui» può avere questa connotazione. È proprio con questa sfumatura peggiorativa che Pirandello usa questi pronomi nelle sue novelle.

- (...) quando costui sempre tutto fremente di calda bestialità festosa, gli s'appressava, (II bottone della palandrana)

- kur ai, gjithë duke u dridhur nga një ndjenjë kafshërie ngazëllyese, i afrohej (Kopsa e setrës, p. 113, «Tregime e novela», Dituria, Tiranë 1999)

- (...) ma s'era veduta così maltrattata da costei, così contrariata anch'essa nei suoi gusti, (...) (Come gemelle)

- (...) por ajo e kishte trajtuar kaq keq, e kishte kundërshtuar aq shumë në punë shijesh (...) (Si dy binjake, p. 121, «Zoja prej dylli», Toena, Tiranë 2010)

Per quanto riguarda la traduzione di questi pronomi dimostrativi toscani bisogna dire che in albanese vengono tradotti con pronomi della lingua standard non trasmettendo così la marcatezza lessicale voluta dall'autore.

Un altro punto interessante viene costituito da alcune forme verbali caratteristiche dell'uso toscano. Le più ripetute sono le forme particolari del presente di alcuni verbi come andare e fare.

- $\quad$ Fo il sensale; e, voi lo sapete: tempacci! (Il fumo)

- $\quad$ Jam sekser dhe ju e dini: kohë të vështira! (Tymi, p. 80, «Jeta lakuriq», Naim Frashëri, Tiranë 1963)

- Sto qua. Ti fo vedere come mi son messa per benino la casetta. (Amicissimi)

- Do ta shohësh sa mirë e kam rregulluar shtëpizën time. (Shokë të ngushtë, f. 69, «Nata e parë», Toena, Tiranë 2008)

Ovviamente anche in questi casi l'uso delle forme dialettali non viene assolutamente percepito da parte del lettore albanese, perché le corrispondenti albanesi delle parole dialettali appartengono alla lingua standard e non ai dialetti.

Nelle novelle di Pirandello si possono individuare anche casi di marcatezza lessicale (caratteristica che fa sì che una parte del testo risalti in confronto al contesto). Pirandello a volte usa nella stessa frase parole che appartengono a registri differenti.

- Uno di quei giorni, finalmente tornò dalla città sconfitto, bocciato, bocciato ancora una volta agli esami di licenza tecnica. E ora basta! basta davvero! non voleva più saperne! Prese libri, quaderni, disegni, squadre, 
astucci, matite e li portò giù, innanzi alla villa per farne un falò. (Scialle nero)

- Një ditë prej ditësh u këthye më në fund nga qyteti, i dërrmuar, i i rrëxuar, i irëxuar edhe një herë në provimet e maturës. Dhe tani mjaft! Mjaft, me gjith mënd! Nuk e bëri të gjatë! Rrëmbeu librat, fletoret, rrigat, mjetet e mësimit, lapsat; zbriti poshtë para vilës për të bërë një zjarr bubulak. (Shalli i zi, p. 32, «Jeta lakuriq», Naim Frashëri, Tiranë 1963)

Mescola termini della lingua parlata bocciato, e della lingua aulica falò. Questa disparità di linguaggio non viene trasmessa nel testo tradotto in albanese. I termini usati appartengono entrambi allo stesso registro dello scritto.

- Va' ad avvertire tua moglie che, a momenti sarà pronto.

- Non ci vado, gnornò! - grugnì Gherlando, pestando un piede. - Andateci voi.

- Spetta a te, somarone! - gli gridò il padre. - Tu sei il marito: va'! (...). I convitati accorsero a metter pace, a persuadere Gherlando a prendere un boccone...

- Ma se non so neppure come debba chiamarla! - gridò Gherlando, esasperato. (Scialle nero)

- Shko e thuaj sat shoqje se buka është gati.

- Nuk vete dot baba! - u përtyp belbët Xherlandi duke përpjekur këmbën - Shko ti.

- Të përket ty, hajvan! - u hakrrua i jati - Ti je burri: çap! (...) Të ftuarit nxituan për të qetsuar ujrat, për t'i pajtuar, për t'i mbushur mendjen Xherlandit t'i bënte zë nuses...

- Nuk di me ç'emër ta thërres! - turfulloi Xherlandi i xhindosur. (Shalli i zi, p. 25, «Jeta lakuriq», Naim Frashëri, Tiranë 1963)

In questa occasione il protagonista si comporta come un contadino: gnornò, dice. Ovvio che la parola debba è una forma troppo corretta e precisa nelle labbra di Gherlando. Anche in questo esempio la marcatezza lessicale non viene trasmessa nel testo tradotto.

\subsection{Termini provenienti dalle altre lingue}

Nell'epoca in cui Pirandello scriveva era costante l'entrata dei forestierismi nella lingua italiana. Pirandello non solo criticò l'imitazione dei classici, ma molto più forte era la sua critica contro l'imitazione degli scrittori stranieri.

La lingua italiana generalmente non adatta il termine straniero al suo sistema fonetico-morfologico: lo lascia com'è. Nella maggioranza dei casi i forestierismi nelle novelle di Pirandello vengono indicati in corsivo oppure con le virgolette. Questa informazione iconica serve al lettore per ricevere in anticipo un'informazione ai fini della comprensione del termine. Uno scrittore dal ricco vocabolario come Pirandello non può non far entrare nelle sue opere molti termini provenienti dalle altre lingue. II termine straniero è molto più frequente in quegli episodi che si situano all'estero.

\subsubsection{Gallicismi}

Tra le altre lingue usate da Pirandello nelle sue novelle i gallicismi sono i più abbondanti. Nelle novelle troviamo esempi di gallicismi adattati e di altri non adattati.

Termini non adattati:

- (...) lo esaminava, lo faceva voltare di qua e di là, Pardon! Pardon! - (...) Marsina stretta

- ...rrinte e vështronte profesorin dhe e kthente sa andej-këndej, Pardon! Pardon! (Fraku i ngushtë, p. 166, «Nata e parë», Toena, Tiranë 2008)

- (...) innanzi allo châlet in Piazza delle Terme (Se...)

- para shalesë së Sheshit të Llixhave (Në rast se..., p. 133, «Teze Mikelina», Toena, Tiranë 2010)

- (...) il proprietario vorrebbe che al bureau si sapesse con precisione (...) Nell'albergo è morto un tale)

- (...) pronari i hotelit kërkonte që në zyrën e informacionit të dinin me saktësi (...) (Në hotel vdiq dikush, p. 133, «Jeta lakuriq», Çabej, Tiranë 1997)

Per dare questi gallicismi i traduttori si riferiscono ai gallicismi usati nella lingua albanese. Così «pardon» è un gallicismo non adattato anche in albanese ed il traduttore lo ha lasciato com'è. «Chalet» è un termine francese che in albanese è stato adattato alle regole fonetico-morfologiche della lingua, invece il termine «bureau» non è stato preso in prestito dall'albanese, perciò il traduttore traduce il termine e non usa il prestito usato da Pirandello.

Termini adattati:

- (...) alla vecchia signora che ingombrava con la sua enorme pinguedine mezzo canapè (Marsina stretta)

- (...) E çuan përpara zonjës së moshuar që kishte zënë gjysmë kanapeje. (Fraku i ngushtë, p. 172, «Nata e parë», Toena, Tiranë 2008) 
- (...) s'era affrettato a disfarsi, tanto di lui, quanto di Corbino, gli ultimi rimasti nella scuderia, per il placido landò della madre (La rallegrata)

- (...) nxitoi dhe i shpërndau kuajt: largoi edhe dy të fundit që patën mbetur në sallë, për llandonin e lehtë të së ëmës. (Kërcim i hareshëm, p. 138, «Jeta lakuriq», Çabej, Tiranë 1997)

In questi ultimi due casi si tratta di parole entrate anche nell'albanese ed adattate alle particolarità della lingua per cui la scelta dei traduttori è stata quella di usare i termini già in uso anche nella loro lingua.

In alcune novelle troviamo la presenza di frasi intere o canzoni in lingua straniera. Nella novella Avemaria di Bobbio il suo protagonista legge per la prima volta il volume «Essais di Montaigne»; concretamente leggeva il capitolo XXVII, dove si dimostra che «c'est folie de rapporter le vrai et le faux à notre suffisance». (Avemaria di Bobbio)

Dopo aver letto alcune righe in francese, Bobbio esclama:

- Eh già! - (...) Eh già! Ce grand sainct Augustin attesta, o diciamo, autentica d'aver veduto, su le reliquie di san Gervaso e Protaso a Milano, un fanciullo cieco riacquistare la vista (Avemaria di Bobbio)

- Posi jo! Ce grand Sainct Augustin na pohoka, apo më mirë na vërtetoka se paska parë përmbi reliktet e shenjtorëve Xhervazio e Protasio, në Milano, një fëmijë të verbër, të cilit i qënkej kthyer të parit (Urata e Bobios, p. 131, «Nata e parë», Toena, Tiranë 2008)

Colto da un grande dolore di denti, cerca di concentrarsi nella lettura del suddetto libro:

- Riprese il libro e la lettura, "une femme nouvellement baptisée lui fit; Hesperius... no, appresso... Ah ecco... une femme en une procession avant touché à la chase Sainct Estienne d'un bouquet, et de ce bouquet s'estant frottée les veux, avoir recouvré la veue qu'elle avait perdue..." (Avemaria di Bobbio)

- pastaj i hapi përsëri. la nisi leximit sërish. "une femme nouvellement baptisée lui fit; Hesperius... no, appresso... Ja këtu... une femme en une procession avant touché à la chase Sainct Estienne d'un bouquet, et de ce bouquet s'estant frottée les veux, avoir recouvré la veue qu'elle avait perdue..." (Urata e Bobios, p. 132, «Nata e parë», Toena, Tiranë 2008)

Nel caso di queste frasi scritte interamente in francese il traduttore ha scelto di ripeterle nella loro lingua originale e per venire incontro alle esigenze di comprensione dei lettori che ignorano il francese con una glossa extratestuale ha dato la traduzione in albanese.

\subsubsection{Iberismi}

Per quanto riguarda l'uso degli iberismi usati da Pirandello nelle sue novelle, bisogna dire che la maggioranza è stata adattata in italiano dal punto di vista grafico, fonetico e morfologico.

Termini non adattati:

- "Un guazzabuglio d'immagini, di ricordi, come in un balenio d'uragano, gli tumultuava nello spirito. La Plata, le pampas (Filo d'aria)

- Një mishmash shëmbëlltyrash dhe kujtimesh i trazonte shpirtin si një shpërthim stuhie. Plata, pampasi (Një fill ere, p. 195, «Nata e parë», Toena, Tiranë 2008)

II traduttore ha scelto di ripetere il termine straniero, però non essendo introdotto anche in albanese questo iberismo risulta una parola strana, incomprensibile. Avrebbe dovuto introdurre una glossa extratestuale con la spiegazione.

Termini adattati:

- le polizzine della riffa costavano un soldo l'una (La Madonnina)

- Biletat e shortit kushtonin një soldë copa. (Zoja prej dylli, p. 36, «Zoja prej dylli», Toena, Tiranë 2010)

- $\quad$ II mezzadro, (...) lui era stato in America, otto anni a Benossarie (Scialle nero)

- bujku (...) kish qenë në Amerikë, tetë vjet në Buenos Aires (Shalli i zi, p. 18, «Jeta lakuriq», Naim Frashëri, Tiranë 1963)

- un vestito grigio d'alpagà (II ventaglino)

- i veshur me tesha të leshta ngjyrë hiri (Erashka, p. 94, «Jeta lakuriq», Naim Frashëri, Tiranë 1963)

- Nane Papa, con le mani grassocce appese alle falde del vecchio pànama sformato, dice a Candelora: (Candelora)

- Nane Papa i vuri duart e majme në rrethin e kapeles së vjetër, kapelë kashte e deformuar, dhe i tha Kandelorës: (Kandelora, p. 123, «Jeta lakuriq», Çabej, Tiranë 1997)

Dopo aver subito le trasformazioni per l'adattamento al sistema fonetico e morfologico della lingua italiana questi termini vanno ad arricchire il lessico dell'epoca ed il lettore li considera termini della propria lingua per cui il corsivo non è 
più necessario. Anche il traduttore albanese li percepisce come termini che appartengono alla lingua italiana, perciò prosegue con la traduzione come tutti gli altri termini.

\subsubsection{Anglicismi}

Gli anglicismi usati da Pirandello appartengono a settori molto concreti, come la moda, i mezzi di comunicazione e la gastronomia. Così come succede anche per i gallicismi, nella maggioranza dei casi si trascrivono i termini tra virgolette 0 in corsivo per comunicare al lettore l'adozione di una certa parola straniera. Si nota l'uso dei termini stranieri in quelle novelle che sono ambientate all'estero oppure che hanno come protagonista personaggi non italiani. Per esempio nella novella /l capretto nero la storia si svolge in Sicilia però ha come protagonista un viceconsole inglese in Girgenti, il sig. Charles Trokley e altri turisti inglesi nell'isola ai quali si riferisce utilizzando i titoli sir e miss.

- Più di tutti lieta e ammirata se ne mostrò, lo scorso aprile, la giovanissima e vivacissima Miss Ethel Holloway. (Il capretto nero)

- Në muajin prill, më e kënaqur dhe më e mrekulluar nga të gjithë u tregua Mis Etel Hallouej (Keci i zi, p. 149, «Tregime e novela», Dituria, Tiranë 1999)

- (...) molto mi compiacqui della gioja di quella piccola Miss. (...) (Il capretto nero)

- (...) aq sa më kënaqi gëzimi i asaj zonjushës së vogël ... (Idem, p. 150)

- (...) Sir W.H. Holloway, ricchissimo e autorevolissimo Pari d'Inghilterra.

- $\quad$ E dërgonte Sër U.H. Halloueji, Lord i Anglisë (Idem, p. 152)

Si tratta di anglicismi conosciuti anche nell'albanese perciò non è stato difficile per il traduttore scegliere la strategia da adottare: usare la forma tipica albanese di questi anglicismi.

\subsubsection{Latinismi}

I latinismi costituiscono un elemento essenziale del lessico di Pirandello come furono anche per altri scrittori come Carducci, D’Annunzio e Pascoli. Lo studio ed il conseguente influsso della lingua e della letteratura latina si può percepire nello stile e nella lingua di Pirandello che appare sempre permeata da numerosi cultismi. In Pirandello dobbiamo distinguere quei latinismi già adattati in italiano che conservano la loro qualità di cultismi indicati talvolta da termini letterari e poetici e gli altri latinismi non adattati che lui usa come espressioni lessicalizzati nella lingua oppure, in altri casi, come litanie con un pizzico di ironia o critica.

Termini non adattati:

- con l'aria di un compitissimo arbiter elegantiarum (Marsina stretta)

- $\quad$ sikur të qe në plot kuptimin e fjalës një arbiter elegantorum (Fraku i ngushtë, p. 166, «Nata e parë», Toena, Tiranë 2008)

- (...) e la fece innamorare coram populo del padre beneficiale Fiorica (La Madonnina)

- dhe e shtyu të binte në dashuri faqe të giithëve me atin Fiorika (Zoja prej dylli, p. 31, «Zoja prej dylli", Toena, Tiranë 2010)

- (...) da cui si fa ajutare nelle cure sui generis che impartisce ai malati (Acqua e li)

- që e ndihmon për t'u bërë të sëmurëve mjekimet sui generis (Ujë dhe pikë, p. 183, «Nata e parë”, Toena, Tiranë 2008)

- Ora me le avrebbe protestate ipso facto, capisci? (Amicissimi)

- Tani do të m'i kërkonte se s'bën! (Shokë të ngushtë, f. 73, «Nata e parë", Toena, Tiranë 2008)

- (...) l'avemaria, questa volta in latino... gratia plena... Dominus tecum... fructus ventris tui... nunc et in hora mortis... (...) Amen (L'Avemaria di Bobbio)

- kësaj radhe duke i thënë fjalët në latinisht... gratia plena... Dominus tecum... fructus ventris tui... nunc et in hora mortis... (Urata e Bobios, p. 132, «Nata e parë", Toena, Tiranë 2008)

La strategia scelta da parte dei traduttori albanesi è la stessa: ripetizione dei termini in latino e per risolvere i problemi di comprensione del lettore albanese hanno dato con una glossa extratestuale la traduzione linguistica in albanese.

Termini adattati:

Per quanto riguarda gli altri latinismi che conservano la qualità dei cultismi bisogna dire che, in alcune occasioni, questo uso sembra esprimere un'intenzione stilistica molto chiara. Troviamo tutta una serie di sostantivi, aggettivi, verbi e avverbi usati con valore letterario o poetico che servono per elevare il tono del contenuto della scrittura. 
1. Verbi: annunziare, guatare, denunziare, garrire (nel significato di "sventolare") avventare, springare, perorare, pollare ecc.

- E ora, ecco, arrivavano le paranze, una dopo l'altra, con le vele che garrivano allegre (La maestrina Boccarmè)

- Dhe ja, ku vinin parancat, njëra mbas tjetrës, me velat që cijatnin gëzueshëm (Mësuesja Bokarme, p. 121, «Tregime e novela», Dituria, Tiranë 1999)

II termine scelto in albanese appartiene al linguaggio letterario riflettendo in una certa misura la scelta stilistica dell'autore.

- Alzò subito gli occhi a guatarla in viso. (Filo d'aria)

- Ngriti menjëherë sytë për ta parë në fytyrë (Një fill ere, p. 195, "Nata e parë», Toena, Tiranë 2008)

- Miss Ethel Holloway commise l'indelicatezza di voltargli le spalle improvvisamente per correr dietro a un grazioso capretto nero, nato da pochi giorni, che tra le capre sdraiate springava qua e là (II capretto nero)

- mis Etel Hallouej u soll pa prekë, aty për aty i ktheu shpinën dhe vrapoi mbas një keci të lezetshëm të zi, që kishte lindur para pak ditësh dhe që, mes dhive të shtrira, kërcente hop këtu e hop aty (Keci i zi, p. 150, "Tregime e novela», Dituria, Tiranë 1999)

Nei casi riportati i cultismi usati dallo scrittore vengono tradotti con termini del linguaggio comune e che non vengono riconoscono per qualche loro uso particolare. In questo modo lo stile dello scrittore non viene trasmesso nel testo tradotto provocando delle perdite nel processo traduttivo.

2. Sostantivi: presidio, oltracotanza, esordio, pervicacia, sacrificio, virgulto, orfanotrofio, doglie, viatico, famiglio, lustro, ferula, gerontocomio, ricetto, convittore ecc.

- (...) ne provava tale gaudio e insieme tale sgomento (La Madonnina)

- ndiente një ngazëllim aq të madh dhe në të njëjtën kohë një tronditje aq të fortë (Zoja prej dylli, p. 35, «Zoja prej dylli», Toena, Tiranë 2010)

- Come, passando per un giardino e allungando distrattamente una mano, si bruca un tenero virgulto e se ne sparpagliano in aria le poche foglioline. (La maestrina Boccarmè)

- $\quad$ Ashtu sikundër ndodh kur njeriu kalon mes një kopshti dhe zgjat dorën i shkujdesur e këput një filiz të njomë e hapërdahen gjethëzat e pakta (Mësuesja Bokarme, p. 121, «Tregime e novela», Dituria, Tiranë 1999)

- Doveva aver fine, perdio, una così enorme sconcezza, una siffatta oltracotanza. (II bottone della palandrana)

- Dreqi e mori, duhej të merrte fund ajo punë e ndyrë, ajo skrutë e paparë. (Kopsa e setrës, p. 116, «Tregime e novela», Dituria, Tiranë 1999)

- Perciò ogni risposta che dava loro era come un colpo di ferula su le dita. (La difesa del Mèola)

- Ndaj dhe çdo përgjigje që u jepte ishte si një fshikullim purteke. (Mbrojtja e Meolës, p. 33, «Nata e parë», Toena, Tiranë 2008)

- ecco è arrivato finanche ad alloggiarli nella sua villetta, per avere l'onore di dar ricetto a un portento dell'arte, (Candelora)

- i cili arrin deri aty sa t'i strehojë në vilën e tij, për të patur nderin të ketë mysafir një yll të artit (Kandelora, p. 126, «Jeta lakuriq», Çabej, Tiranë 1997)

Alcuni dei termini usati nella traduzione appartengono al linguaggio letterario, altri appartengono al linguaggio comune. Quindi a volte viene rispettata l'intenzione dell'autore e altre volte, invece, no.

3. Aggettivi: ubertoso, diuturno, cospicuo, subitaneo, inviso, discinta, fulvo, superno, blando, cogitabondo, famigerato, brullo, munifico, miserando, specioso, nefando, silente ecc.

- e non rifinivano di porgli sotto gli occhi e di fargli toccar con mano le miserande piaghe della loro esistenza (I fortunati)

- dhe s'lodheshin duke treguar haptaa varrat e jetës së tyre mierane (Fatlumët, p. 42, «Nata e parë», Toena, Tiranë 2008)

- dei tradimenti operati da essi negli anni nefandi della tirannia borbonica (La difesa del Mèola) (Mbrojtja e Meolës, p. 29, «Nata e parë», Toena, Tiranë 2008)

- dhe tradhtinë që kishin bërë ata gjatë viteve të rënda të tiranisë së Burbonëve

- Quel dondolio lento di code prolisse, quel raspare di zoccoli, di tratto in tratto, certo erano di cavalli cogitabondi. (La rallegrata)

- $\quad$ Ajo lëkundje e lehtë e bishtave të gjatë, ajo e rrëmihur e tokës me këmbë, kohë pas kohe, ishin shenja e 
gjeste kuajsh të kredhur në mendime. (Kërcim i hareshëm, p. 139, «Jeta lakuriq», Çabej, Tiranë 1997)

- d'una bellezza a cui i diuturni dolori avevano dato la grazia d'una soavissima mestizia (II bottone della palandrana)

- një bukuri së cilës hallet e niëpasniëshme i kishin mveshur një hir pikëllimi gjithë nur (Fraku i ngushtë, p. 169, «Nata e parë», Toena, Tiranë 2008)

- Basta questo, perch'a Milocca sia inviso a tutti: inviso per principio (Acqua e lì)

- Mjafton kjo që në Miloka ta shohin të gjithë shtrembër, ashtu, për parim, pa mbajtur parasysh rezultatet e punës së tij (Ujë dhe pikë, p. 183, «Nata e parë», Toena, Tiranë 2008)

- (...) coi magnifici capelli fulvi scomposti intorno al bel volto pallido (Come gemelle)

- me flokët e mrekullueshëm kuqërremtë të shkrehur rreth e rreth fytyrës së zbehtë (Si dy binjake, p. 120, «Nata e parë», Toena, Tiranë 2008)

I cultismi in questi casi vengono tradotti con termini che appartengono alla lingua scritta, non necessariamente letteraria e così non riescono a trasmettere al lettore albanese questa particolarità della lingua di Pirandello.

4. Altri termini: iniquamente, vie più, finanche, lunghesso ecc.

- (...) e lunghesso il canale quell'eleganza di gracili salci violetti e tanto dolce azzurro l'ombra che dilaga (II vecchio Dio)

- (...) gjatë vijës së ujit një hijeshi shelgjesh të brishtë me fletë si në ngjyrë të manushaqes dhe një tis të gjerë kaltërsie të ëmbël që shtrihej si liqen (Zoti i vjetër, p. 52, «Jeta lakuriq», Çabej, Tiranë 1997)

- Ma no, Giorgio! - gemette allora lei, raffondando vie più il capo e torcendosi in grembo le mani. - No! non è possibile! non è possibile! (Scialle nero)

- Jo Xhorxh, jo! - rënkoi atëherë ajo duke ulur edhe më kokën dhe duke i shtrënguar duart në prehër. - Jo, nuk është e mundur, nuk është punë që bëhet! (Shalli i zi, p. 20, «Jeta lakuriq», Naim Frashëri, Tiranë 1963)

- $\quad$ Non si doveva darla vinta al destino, che favoriva così iniquamente l'ipocrisia di tutti quei signori radunati nell'altra stanza! (Marsina stretta)

- $\quad$ Nuk duhej t'i dorëzohej fatit që i kishte dalë në krah kaq padrejtësisht hipokrizisë së gjithë atyre zotërinjve që ishin mbledhur në dhomën tjetër. (Fraku i ngushtë, p. 175, «Nata e parë», Toena, Tiranë 2008)

Anche in questi casi la scelta di parole di uso comune nella traduzione non rispetta lo stile e la scelta dello scrittore del testo originale.

\section{Conclusioni}

In questo studio abbiamo preso in analisi la traduzione in albanese del linguaggio delle novelle di Pirandello e le difficoltà che sorgono durante la traduzione nonostante l'impressione che si tratti di un linguaggio semplice che non pone problemi di sorta. Oggetto di studio sono stati i segmenti testuali in dialetto e quelli appartenenti ad altre lingue. Dopo l'analisi di alcune delle sue novelle tradotte in albanese concludiamo lo studio nel seguente modo:

- la strategia predominante nella traduzione del dialetto è quella della traduzione del dialetto con l'albanese standard. II testo tradotto si è allontanato così dal testo originale, dal codice artistico dell'autore, e si è avvicinato all'individualizzazione espressiva del traduttore evidenziando così l'idioletto espressivo del traduttore.

- per la traduzione dei segmenti linguistici in altre lingue (gallicismi, anglicismi) i traduttori hanno seguito la strategia della ripetizione in alcuni casi per i termini non adattati e la strategia della traduzione nel caso dei termini adattati.

- per la traduzione dei latinismi non adattati la ripetizione e la glossa extratestuale per spiegare il termine pare essere la scelta principale dei traduttori; per i latinismi adattati, invece, la strategia principale è quella della traduzione. essendo dei termini che costituiscono dei cultismi servono allo scrittore per creare cambiamenti di tono nel discorso, questi cambiamenti appaiono trasmessi nei testi tradotti con maggiore o minore intensità.

Cercando di osservare l'equivalenza funzionale o skopos theory (una traduzione deve produrre lo stesso effetto a cui mirava l'originale) possiamo dire che nel caso della traduzione della lingua di Pirandello ci troviamo di fronte a delle perdite nel processo traduttivo. 


\section{References}

Pirandello, L. (1963). Jeta lakuriq (La vita nuda), Tirana: Naim Frashëri.

(1997). Novela për një vit. Jeta lakuriq (La vita nuda), Tirana: Çabej.

(1999), Tregime e Novela (Racconti e novelle), Tirana: Dituria.

(2008). Nata e parë (Prima notte), Tirana: Toena.

, (2008). Zoja prej dylli (La Madonnina), Tirana: Toena.

(2010). Teze Mikelina (Zia Michelina), Tirana: Toena.

Blanco, A. N. (1990). La lengua y el estilo di Luigi Pirandello, tesis doctoral.

Carbonell I Cortes, O. (1999). Traducción y cultura: de la ideología al texto, Madrid: Ediciones Colegio de España.

Catfort J. C. (1965). A Linguistic Theory of Translation, An Essay in Applied Linguistics, Language and language learning, London: Oxford University Press.

Dardano, M.\&Trifone P. (1997). La nuova grammatica della lingua italiana, Bologna: Zanichelli Editore.

Lefevere, A. (1992). Translating Literature: Practice and Theory in a Comparative Literature Context, New York: The Modern Language Association of America.

(1997). Traducción, reescritura y la manipulación del canon leterario, Ma Carmen Africa Vidal dhe Román Alvarez (përkthyes). Salamanca: Ediciones Colegio de España (Translation, Rewriting and the Manipulation of Literary Fame, Londër: Routledge, 1992).

Lo Vecchio-Musti, M. (a cura di) 1960. Saggi, poesie e scritti varii, Milano: Mondadori.

Momigliano, A. (1960). Storia della letteratura italiana, Principato.

Newmark, P. (1981) Approaches to Translation, Oxford: Pergamon Press. , (1987) A textbook of translation, London: Prentice Hall.

Papa, M. (2012). Translating dialect: a comparative study of the novels of Luigi Pirandello, in «Mediterranean Journal of Social Sciences» Vol. 3 Nr. 2, publisher Mediterranean Center of Social and Educational Research, fq. 413-422, Roma, Italia, ISSN 2039-9340.

Puglisi, F. (1968). "Il dialetto siciliano nella lingua di Pirandello", in Pirandello e la sua lingua, Cappelli Editore.

Rabadan, R. (1994). Traducción, intertextualitad y manipulación, tek Amparo Hurtado Albir (ed.) Estudis Sobre la traducció, Castelló, Universitat Jaume I, 129-39. 